\title{
Auto-Sapiens Autonomous Driving Vehicle
}

\author{
Maicol Laurenza, Gianluca Pepe and Antonio Carcaterra \\ Department of Mechanical and Aerospace Engineering of Sapienza University of Rome, Italy
}

Keywords: Autonomous Car, Collision Avoidance, Velocity Obstacle, Optimal Feedback Control.

\begin{abstract}
This paper presents the Auto-Sapiens project, an autonomous driving car developed by the Mechatronics and Vehicle Dynamics Lab, at Sapienza University of Rome. Auto-Sapiens is a technological platform to test and improve innovative control algorithms. The car platform is a standard car (Smart ForTwo) equipped with throttle, brake, steering actuators and different sensors for attitude identification and environment reconstruction. The first experiments of the Auto-Sapiens car test a new obstacle avoidance. The vehicle, controlled by an optimal variational feedback control, recently developed by the authors, includes the nonlinearities inherent in the car dynamics for better performances. Results show the effectiveness of the system in terms of safety and robustness of the avoidance maneuvers.
\end{abstract}

\section{INTRODUCTION}

Autonomous driving is a challenging integrated technology associated to the benefits for the people's life quality, safety, reduction of accidents and traffic. The Society of Automotive Engineers - SAE defined the automation levels of a vehicle (A. Taeihagh and H. S. M. Lim, 2019), describing to what extent an automated driving system ranging from auxiliary assistance, up to excluding the driver completely. One of the important issues is accident management. An automated vehicle must be able to drive in the presence of external disturbances such as unautomated vehicles or careless pedestrians, passing animals, etc. This can result in a wide range of possible accident cases that an automated Level 4 system must be able to deal with, making important decisions to avoid the crash.

This paper is devoted to the development of level 3-4 automated driving systems, in which even if the driver is careless or absent-minded, the vehicle manages to avoid obstacles. To date, many car manufacturers advertise Advanced Driver-Assistance Systems (ADAS) for obstacle avoidance without, however, any information on how algorithms work. For these reasons, the Auto-Sapiens project (Antonelli et al., 2018, 2019a, 2019b; Laurenza et al., 2019; Pepe et al., 2019) aims at developing and testing an autonomous driving system original for its mathematical formulation and technological implementation.
A collision avoidance system acts on two separate levels: (i) perception or identification of a possible accident, (ii) definition of a new path to follow. Perception is managed by several proprioceptive and exteroceptive sensors for estimating the state of the vehicle, identifying obstacles and free space around the vehicle, and eventually recognizing road markings as driving directions, pedestrian crossings, crossroads, etc. The onboard controllers manage the information obtained by the sensors recognition. Through a path-generator, it defines the final vehicle maneuver.

Typical sensors of an autonomous vehicle are: high-resolution cameras, radar, LIDAR, ultrasonic sensors for the estimation of the surrounding environment, and satellite-based systems such as Global Position System (GPS), Inertial Measurement Unit (IMU), odometry, Wireless Wide Area Networks (WWANs) such as 3G/4G/5G or Wi-Fi for the relative positioning. Fusion of heterogeneous information can provide detailed information for the simultaneous localization and mapping (SLAM) of the vehicles and obstacles (Song et al., 2019). In fact, it is very common to achieve a better estimation of the vehicle's position by using data fusion techniques such as Bayesian filtering and Kalman Filters. Moreover, the next-generation ADAS will increasingly use wireless network connectivity to offer improved value by using vehicle-to-vehicle (V2V) and vehicle-to-infrastructure (V2X) data (Ullah et al., 2020). Finally, road markings can be 
detected from cameras that identify road signs and often merge information from databases of updated road maps such as Open Street Map (www.openstreetmap.org; Jian et al., 2019).

Once the vehicle obtains information on the surrounding environment, the planning step produces the optimal trajectory to navigate safely to the desired destination, according to sensed data. In the event of a very fast emergency maneuver, since the optimal trajectory generator requires a considerable amount of computational time, often the preferred maneuver is the simple braking through the Advanced Emergency Braking System (AEBS).

For these reasons, the authors propose a new obstacle avoidance method based on the Velocity Obstacle (VO) method already investigated in (Pepe et al., 2019; Laurenza et al., 2019) and which, for the first time, is implemented on the real vehicle AutoSapiens. The first experiments aim to analyze how well the vehicle reacts to different accident scenarios, and in the presence of a non-controlled vehicle.

Section 2 describes the vehicle equipment fitted with on-board computers actuators and sensors. Section 3 describes how the driving and control algorithm works. Section 4 examines the first tests of accident evasion maneuvers.

\section{AUTO-SAPIENS ARCHITECTURE}

The autonomous vehicle of the Mechatronics and Vehicle Dynamics Lab, at Sapienza University of Rome, named Auto-Sapiens is a Smart ForTwo CityCoupe suitably modified. This section describes the overall architecture of the vehicle and the hardware changes to transform it into an autonomous platform. The choice of a Smart ForTwo is due to the interest in creating a compact technological platform to be installed on every vehicle, also on small size ones.

\subsection{Electronic Board and Sensors}

The guidance and control system of the car is shown in Figure 1 e 2. The central control unit is the Zynq by Xilinx based on ARM Dual-Core Cortex-A9. The board has a different digital IO physical connection as I2C, P-MOD, USB 2.0, Gigabit Ethernet and CanBus. This type of board has the advantage of being able to work both in the hardware in the loop with Matlab $\odot$ Simulink $\odot$ and being able to transfer the code directly to FPGA by HDL Coder, allowing the achieving of maximum hardware performances. This allows producing fast numerical codes through Matlab( Simulink $($, taking advantage of many ready-to-use and easy-to-use applications.

The sensor equipment includes a 9DOF inertial platform ST ASM330LHH, GPS SKYTRAQ S1216F8-BD module, four wheels encoders, a LIDAR Velodyne, long-range radar AWR1243 and short-range radar AWR1642, and ultrasonic sensors MB7040-200 Maxbotix (Figure 3). The board is also connected to the car Can-bus to read data from OBD2. The outputs of the Zynq's control unit are all Can-bus i.e. the steering, brake and gas actuators. The gearbox is automatic and is controlled by the original car's control unit ECU.

Dealing with environment reconstruction is not the actual main goal of the project, so the identification of the obstacle is performed by Vehicle To Vehicle $(\mathrm{V} 2 \mathrm{~V})$ communication in which the obstacle sends its position and attitude to the controlled vehicle (Figure 1). This allows us to focus only on the analysis of the performances of the control algorithm and actuator management.

\section{Controlled vehicle}

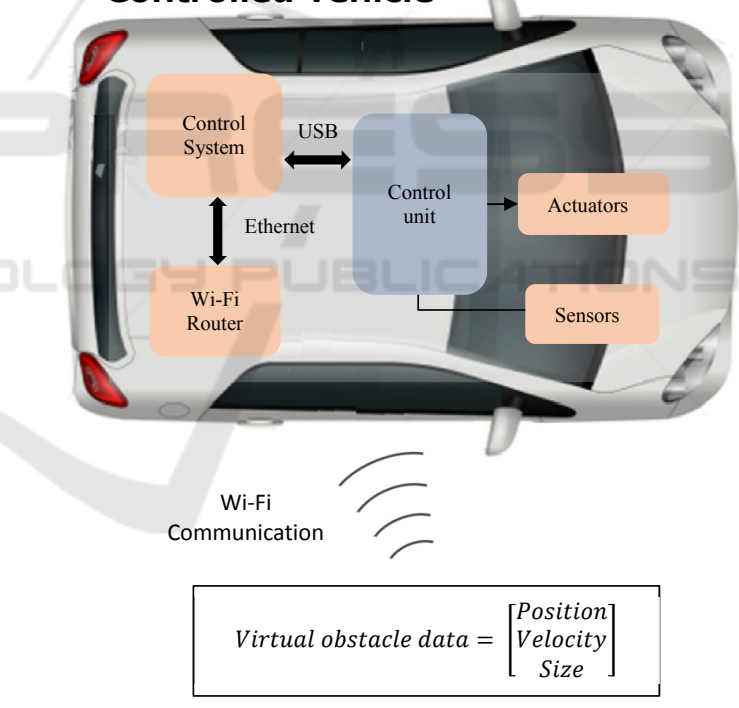

Figure 1: Autonomous driving architecture.

In future developments, the localization of the obstacle will be performed by the combination of radar, lidar and camera data.

The first version of the vehicle is led by a control system (PC) running on Matlab/Simulink software as an initial attempt of hardware in the loop before writing the code on the hardware itself. The PC is connected to the central unit with USB port, sending and receiving data at $20 \mathrm{~Hz}$, and local network receiving data from the obstacle through UDP wireless communication at $30 \mathrm{~Hz}$. 


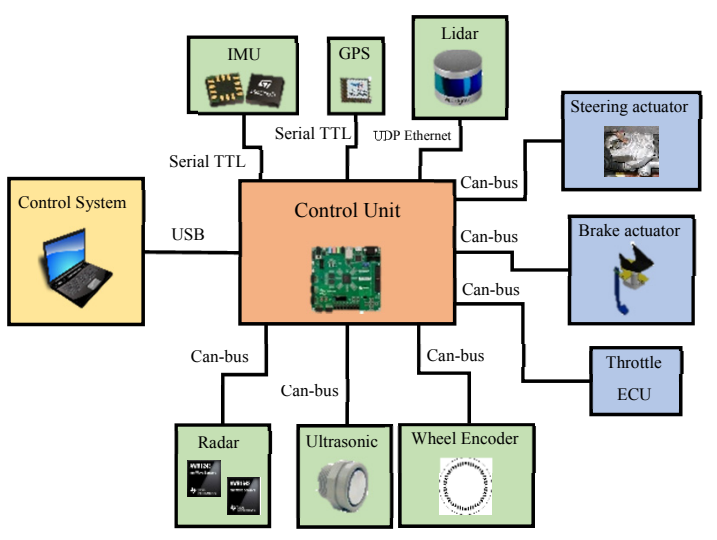

Figure 2: Hardware connection scheme.

The vehicle is equipped with a complete set of actuators and manual stop exclusion systems, both from inside the vehicle and via radio signals at $2.4 \mathrm{GHz}$. In addition, a safety protocol forces the vehicle into a locked state when data transmissions from the obstacles show some failures.

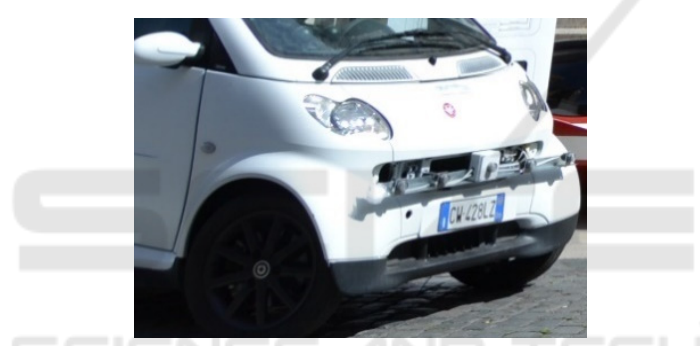

Figure 3: Radar and ultrasonic sensors installed on the front of the vehicle.

For the localization and attitude identification of the vehicle, the GPS, IMU and odometry are used.

The GPS works at a maximum frequency of $50 \mathrm{~Hz}$ with an accuracy of $2.5 \mathrm{~m}$ CEP for position, $0.1 \mathrm{~m} / \mathrm{s}$ for velocity.

The IMU has an update frequency of $100 \mathrm{~Hz}$ and has a full-scale acceleration range up to $\pm 16 \mathrm{~g}$, but for our purposes the actual range is $\pm 2 \mathrm{~g}$, and a wide angular rate range from \pm 125 of $\pm 4000 \mathrm{dps}$ (degrees per second) that enables its usage in a broad range of automotive applications.

Odometry is used to locate the vehicle during the motion, measuring the angular velocity of the wheels. In this case, the velocity of the wheels is taken from the ABS system, which has sensor rings with 42 number of teeth.

In Figure 4 is shown the overall architecture of the autonomous kit. In front of the passenger seat, the control unit is settled, to which the main control system is connected. The only visible actuator is the braking one, which is directly installed on the pedal.

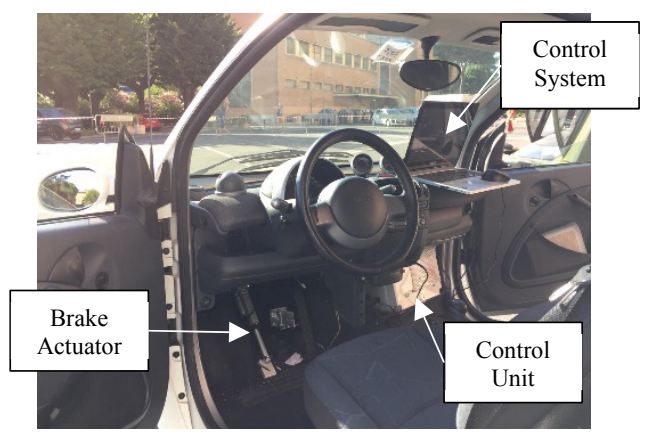

Figure 4: Autonomous kit on the Auto-Sapiens platform.

\subsection{Actuators}

The standard vehicle has been prepared to integrate two motor actuators, the steering and the brake and one electronic actuator, the throttle. The first one is the steering actuator which is the electric power steering, with its torque sensor. The car features rackand-pinion steering, like Figure 5 shows, and the part is connected directly to the steering pinion in series, so that the manual control $\mathrm{M}$ is disabled when the actuator Mc is working.

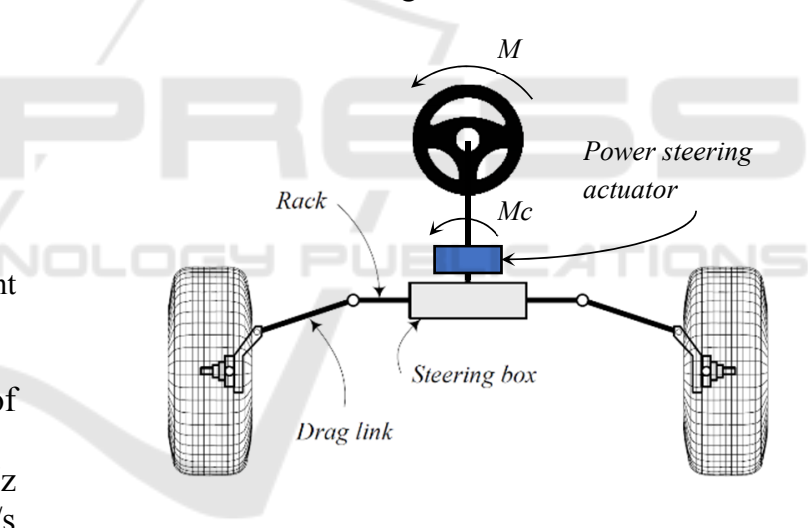

Figure 5: Steering actuator scheme.

To measure the rotation angle of the steer, a sensor has been set directly on the steering wheel (Figure 7).

The second motor actuator is the brake system (Figure 4). It is controlled by a linear actuator with a $20 \mathrm{~mm}$ linear stroke, driven by a DC motor. This linear motor is the real device that applies the pressure of the brake pedal. It is positioned under the steering wheel and in front of the rider's knees, so as not to hinder the rider's legs. This device allows the use of the brake pedal by the pilot even with the actuator installed. It has an axial thrust force of $70 \mathrm{Kg}$ and its purpose is to reproduce the pressure of the brake pedal by the operator, but automatically. 


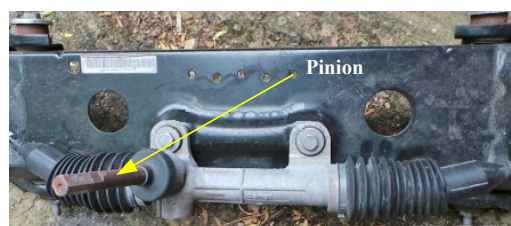

a)

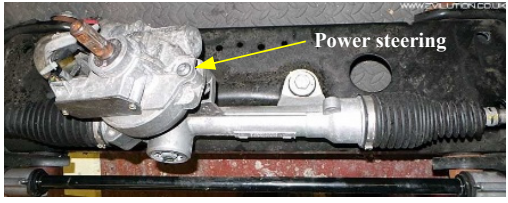

b)

Figure 6: Steering gear before (a) and after (b).

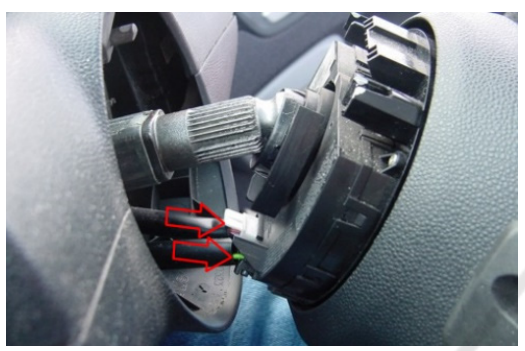

Figure 7: Steering angle sensor.

The throttle is completely electronic and can be controlled via ECU. Two potentiometers were already present to evaluate the position of the pedal through the ECU.

\section{CONTROL SYSTEM}

The control system architecture is represented in Figure 8 in which the nonlinear optimal control algorithm manages the input control $\boldsymbol{u}$ of the car to follow an imposed state target $\boldsymbol{x}_{t}$ through the minimization of an objective function $E\left(\boldsymbol{x}_{t}\right)$. With the engine and steering model, the input control is modified to the one required by the real car, which are the steering wheel angle, throttle and brake percentage.

Once the obstacle data from the V2V infrastructure is received, the decision-making control analyses if there is a crash case and can activate the obstacle avoidance control algorithm instead of the standard path following control.

The main physical and geometrical properties of the car were experimentally measured or have been supposed where measurements were not possible.

The values are listed in Table 1. The car parameters are used to create a dynamic model to assist the nonlinear optimal control, explained below.

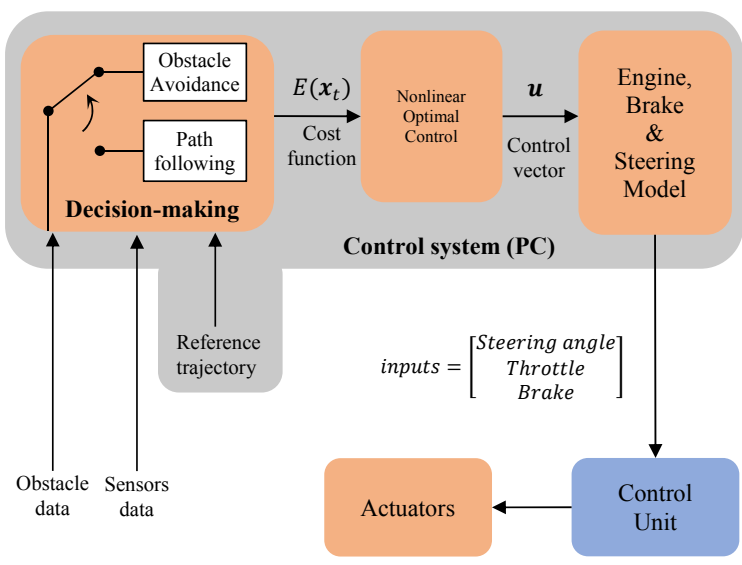

Figure 8: Control diagram.

Table 1: Car parameters.

\begin{tabular}{|c|c|}
\hline Parameters & Values \\
\hline Mass & $950 \mathrm{Kg}$ \\
\hline Yaw Inertia (supposed) & $2000 \mathrm{Kg}^{*} \mathrm{~m}^{\wedge} 2$ \\
\hline Wheelbase & $1.83 \mathrm{~m}$ \\
\hline $\begin{array}{c}\text { Distance between front } \\
\text { wheel and CoG }\end{array}$ & $1.03 \mathrm{~m}$ \\
\hline $\begin{array}{c}\text { Distance between rear } \\
\text { wheel and CoG }\end{array}$ & $0.8 \mathrm{~m}$ \\
\hline Track & $1.24 \mathrm{~m}$ \\
\hline Wheel radius & $0.2 \mathrm{~m}$ \\
\hline $\begin{array}{c}\text { Wheel inertia } \\
\text { (supposed) }\end{array}$ & $1 \mathrm{Kg} \mathrm{m}^{\wedge} 2$ \\
\hline Max torque & $92 \mathrm{Nm}$ at $4500 \mathrm{rpm}$ \\
\hline Max Power & $52 \mathrm{~kW}$ at $5800 \mathrm{rpm}$ \\
\hline 0 - $100 \mathrm{~km} / \mathrm{h}$ & $15.5 \mathrm{~s}$ \\
\hline Steering ratio & $22: 1$ \\
\hline
\end{tabular}

\subsection{Nonlinear Optimal Control}

The authors have developed a new control algorithm based on the optimal control theory, named Feedback Local Optimality Principle - FLOP, which has been tested in simulation environments for different cases (Antonelli et al., 2019a, 2019b; Pepe et al., 2019; Laurenza et al., 2019; Pepe et al., 2018). The algorithm belongs to the class of the variational controls and the problem statement is to minimize a $J$ cost function shown in the following equation:

$$
J=\int_{0}^{\infty} E(\boldsymbol{x}, \boldsymbol{u})+\lambda^{T}(\dot{\boldsymbol{x}}-(\boldsymbol{\phi}(\boldsymbol{x})+\boldsymbol{B} \boldsymbol{u})) d t
$$

The objective function $E(\boldsymbol{x}, \boldsymbol{u})$ is of the type of $\frac{1}{2} \boldsymbol{u}^{T} \boldsymbol{R} \boldsymbol{u}+g(\boldsymbol{x})$. The $\boldsymbol{u}$ and $\boldsymbol{x}$ are the control and state vector respectively and $g(\boldsymbol{x})$ is any penalty function derivable in the state while $\frac{1}{2} \boldsymbol{u}^{T} \boldsymbol{R} \boldsymbol{u}$ is a quadratic penalty function for the control. The dynamic equation is of the type of $\dot{\boldsymbol{x}}=\boldsymbol{\Phi}(\boldsymbol{x})+\boldsymbol{B u}$, where is 
nonlinear state dependent. Thanks to the new formulation is possible to obtain a feedback control law (Pepe et al., 2018):

$$
\boldsymbol{u}=\boldsymbol{R}^{-T} \boldsymbol{B}^{T}\left[\nabla_{x} \boldsymbol{\phi}^{T}-\frac{1}{\Delta \tau} \boldsymbol{I}\right]^{-1} \nabla_{x} g
$$

where $\nabla_{x} g$ is the derivative of the generalized penalty function $g(\boldsymbol{x})$ and $\nabla_{x} \boldsymbol{\phi}$ is the derivative of the nonlinear part of the dynamic equation.

The dynamic model used for the control algorithm is the bike model (Laurenza et al., 2019) depicted in Figure 9. It has 5-degrees of freedom: longitudinal and lateral velocity $u, v$ respectively, yaw rate $\omega$, rotational speed of frontal wheel $\omega_{f}$ and rear wheel $\omega_{r}$ in the mobile reference frame. It has 2-degrees of control: steering $\delta$, rear $C$ torque. The state vector is composed by the fixed reference frame position $\boldsymbol{\eta}=$ $[X, Y, \psi]$ and the 5-degrees of freedom $\boldsymbol{v}=$ $\left[u, v, \omega, \omega_{r}, \omega_{f}\right]$. The equations of the model are:

$$
\begin{gathered}
M \dot{v}+C(v) v=\tau \\
\dot{\eta}=J(\psi) v
\end{gathered}
$$

In eq. (3) $\boldsymbol{M}$ is the inertia matrix, $\boldsymbol{J}$ is the rotational matrix and $\boldsymbol{\tau}$ are the external forces. These are composed of contact forces and external disturbances.

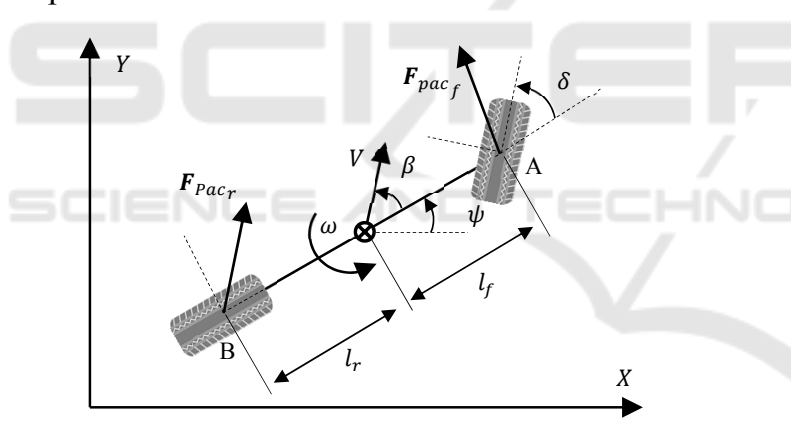

Figure 9: Bike model.

The contact forces $\boldsymbol{F}_{p a c_{f}}$ and $\boldsymbol{F}_{\text {pac }}$ are modeled by the non-linear Pacejka model, which considers a mutual dependence of the longitudinal and lateral slip ratios and a linear dependence with the normal forces acting on the wheels. As for the external disturbances, the rolling resistance and aerodynamic forces are modelled as quadratic functions of the longitudinal speed $u$.

\subsection{Decision-making Control}

As stated in section 3, two different control strategies are chosen by the decision-making control algorithm: the path following or the obstacle avoidance strategies. Depending on the case, the control chooses to use different objective functions $g(\boldsymbol{x})$ of eq. (2) explained below.

\subsubsection{Path Following Strategy}

When the vehicle doesn't identify any obstacle, the path following strategy is enabled through the definition of a penalty function $g_{\text {Path }}(\boldsymbol{x})$.

$$
\begin{gathered}
g_{\text {Path }}(\boldsymbol{x})=g_{1}(\psi)+g_{2}(\omega)+g_{3}(u, v) \\
+g_{4}(X, Y, \psi)
\end{gathered}
$$

with

$$
\begin{aligned}
& g_{1}(\psi)=\frac{1}{2} q_{1}\left(\psi-\psi_{t}\right)^{2} \\
& g_{2}(\omega)=\frac{1}{2} q_{2} \omega^{2} \\
& g_{3}(u, v)=\frac{1}{2} q_{3}\left(V-V_{t}\right)^{2} \\
& g_{4}(X, Y, \psi)=\frac{1}{2} q_{4} e^{2}
\end{aligned}
$$

The $q_{i}$ are tuning parameters to control the yaw $\psi$, yaw rate $\omega$, longitudinal speed $u$ and reduce the distance $e$ with the trajectory. The target points are two different ones: i) $P$ is the closest point to the center of gravity of the vehicle from the desired trajectory, through which we can calculate the lateral offset $e$; ii) $P_{h}$ is the point on the path ahead of $P$ by the parameter $h$, which is the preview distance through which we can set up the incoming maneuver (see Figure 10). This parameter is a tuning one and lets you decide how much you want the vehicle to anticipate the maneuver to better follow the trajectory, due to the delay of actuators and sensors. The $g_{2}(\omega)$ is used to soften the angular velocity. The velocity of the vehicle $V=\sqrt{u^{2}+v^{2}}$ is controlled in terms of the velocity target $V_{t}$, instead the direction in terms of the yaw target $\psi_{t}$, both evaluated in $P_{h}$.

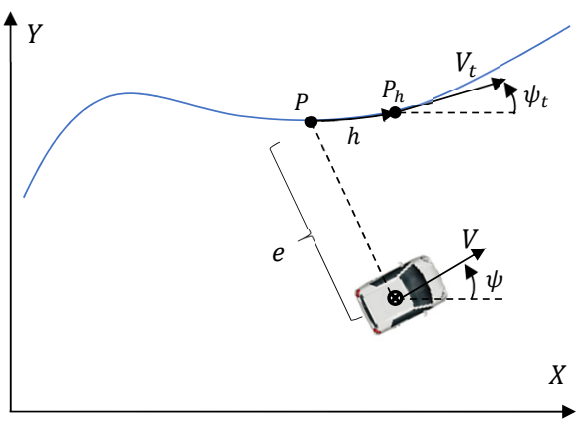

Figure 10: Path following strategy. 


\subsubsection{Obstacle Avoidance Strategy}

If an obstacle is detected during the motion, the obstacle avoidance technique is employed. The method developed by the authors is described in (Laurenza et al., 2019) and is based on the velocity obstacle approach. This specific procedure allows identifying, for the vehicle, an unsafe region of velocities that will cause future crashes with other obstacles (Figure 11).

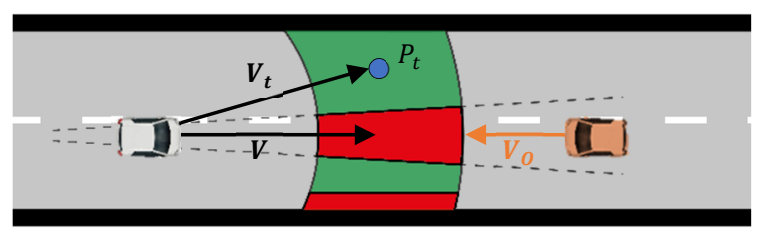

Figure 11: Obstacle avoidance strategy.

To lead the vehicle in a safe state, we defined a penalty function $g_{O A}(\boldsymbol{x})$ that moves the velocity to the center $P_{t}$ of the green area (Figure 11), which is the largest region of the safest velocities, considering the boundary of the road.

$$
\begin{aligned}
& \qquad g_{O A}(\boldsymbol{x})=g_{1}(\psi)+g_{2}(\omega)+g_{3}(X, Y, \psi) \\
& \text { with } \\
& g_{1}(\psi)=\frac{1}{2} k_{1}\left(\psi-\psi_{T}\right)^{2} \\
& g_{2}(\omega)=\frac{1}{2} k_{2} \omega^{2} \\
& g_{3}(X, Y, \psi)=\frac{1}{2} k_{3}\left(V-V_{t}\right)^{2}
\end{aligned}
$$

Here the direction is controlled by $\psi_{T}=$ $\operatorname{atan}\left(\frac{P_{t_{y}}}{P_{t_{x}}}\right)$ and the $V_{t}$ is given by the velocity obstacle method (see reference (Laurenza et al., 2019)). Lastly the $k_{i}$ are tuning parameters.

\subsection{State Estimation}

To perform the first tests of vehicle control, a simplified technique has been developed to estimate the state of the vehicle in terms of position, heading and speed. These are the inputs necessary for the control logic to be used by the controller FLOP (see eq. (3). The state vector of the bike model $\boldsymbol{x}=$ $\left[X, Y, \psi, u, v, \omega, \omega_{r}, \omega_{f}\right]$ is estimated by two measures: GPS, from which we take the absolute speed $V$ of the vehicle, and encoders to obtain the angular velocity of the wheels $\omega_{r}$ and $\omega_{f}$.

Through the Bicycle Kinematic Model (Lynch and Frank, 2017) is possible to reconstruct vehicle motion. Equation (6) briefly describes the kinematic differential equations able to estimate the state of the vehicle (Figure 9).

$$
\begin{gathered}
\dot{X}=V \cos (\psi+\beta) \\
\dot{Y}=V \sin (\psi+\beta) \\
\dot{\psi}=\frac{V \cos (\beta) \tan (\delta)}{l_{r}+l_{f}}
\end{gathered}
$$

where

$$
\beta=\operatorname{atan}\left(\frac{l_{r} \tan \delta}{l_{r}+l_{f}}\right)
$$

Thus, knowing the initial conditions and given the speed $V$ and steering $\delta$ inputs, the state can be easily reconstructed, ensuring good accuracy for short acquisition times, that is short distances and vehicle driving at low speeds.

The dedicated software in Figure 12 allows to geo-locate the map with the exact position of the vehicle before starting with the data acquisition.

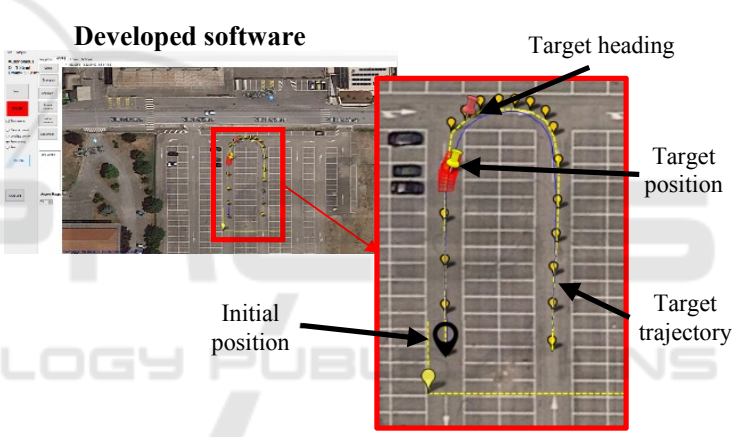

Figure 12: Setting of the target trajectory (yellow waypoints) through developed software that works with maps. The yellow pin is the real-time target waypoint for position, the red one is the real-time target waypoint for heading.

\subsection{Control Inputs}

The central unit requires the percentage of throttle and brake pedal, and angle of the steering wheel as inputs for the control of the actuators.

To manage the acceleration torque, an empirical model for the torque engine has been developed to convert the outputs of the feedback control into the required ones. In Table 2 are shown the transmission ratios used to model the gearbox. 
Table 2: Transmission ratios.

\begin{tabular}{|c|c|}
\hline Ratio $(\boldsymbol{\tau})$ & Values \\
\hline $1^{\circ}$ & $3.37: 1$ \\
\hline $2^{\circ}$ & $2.45: 1$ \\
\hline $3^{\circ}$ & $1.76: 1$ \\
\hline $4^{\circ}$ & $1.33: 1$ \\
\hline $5^{\circ}$ & $0.97: 1$ \\
\hline $6^{\circ}$ & $0.7: 1$ \\
\hline Final & $2.8: 1$ \\
\hline
\end{tabular}

The engine torque curve is evaluated based on Table 1:

$$
\text { Torque }=p 1+p 2 r p m+p 3 r p m^{2}
$$

$$
p 1=\frac{P_{\max }}{r p m_{\max }} ; p 2=\frac{P_{\max }}{\left(r p m_{\max }\right)^{2}} ; p 3=\frac{P_{\max }}{\left(r p m_{\max }\right)^{3}}
$$

where $P_{\max }$ is the max power and $r p m_{\max }$ are the rpm at which the engine achieve the max power.

Figure 13 represents the torque of the engine varying with the throttle percentage. In Figure 14 there is the wheel torque at full throttle with different gear ratios, based on the values in Table 2 .

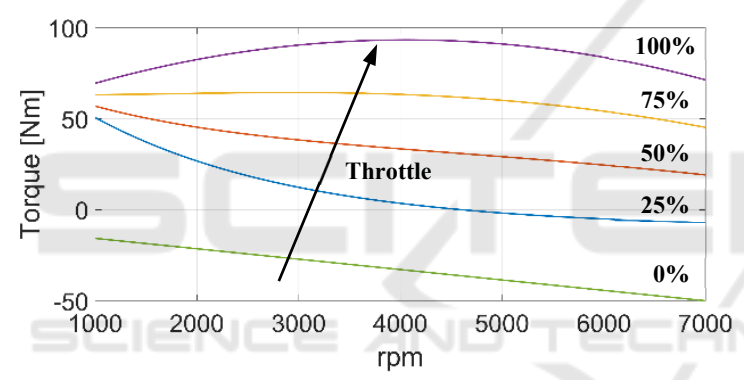

Figure 13: Engine torque varying with throttle.

Reading gear ratio and engine rpm from ECU allows to define the gas percentage to assign to ECU itself. In case that a braking torque is requested by the controller, the braking actuator is engaged to reach the desired torque. Even for the braking actuator, an empirical linear relationship between braking torque and percentage of the pedal has been defined.

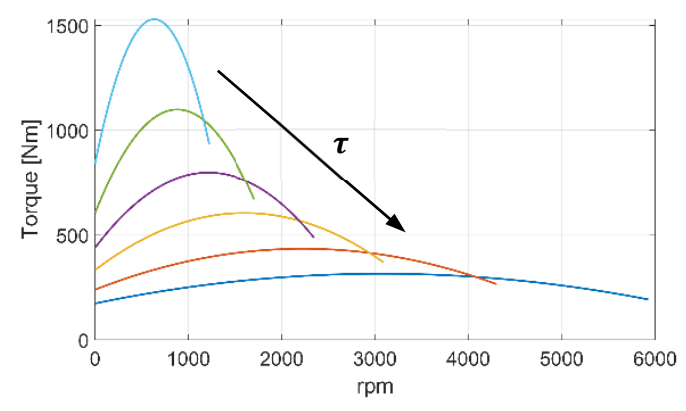

Figure 14: Torque with different gear ratios.
For the steering wheel, given the angle for the inner wheel of the bike model, we use the steering ratio from Table 1 to compute the corresponding target angle for the steering wheel.

\section{EXPERIMENTAL RESULTS}

The tests were performed in a controlled environment and involve the analysis of a frontal crash scenario with a virtual obstacle, which moves at a constant speed as shown in Figure 15.

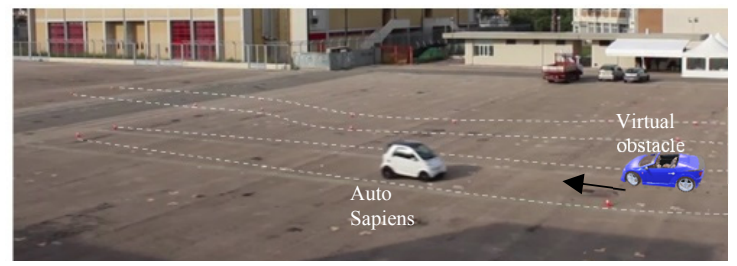

Figure 15: First test of the autonomous vehicle of Sapienza University of Rome, Auto Sapiens.

The performance of the controlled vehicle is tested with velocities belonging to the range of 30-50 $\mathrm{Km} / \mathrm{h}$ and here is shown the test at the max speed of $50 \mathrm{Km} / \mathrm{h}$. The vehicle has to follow a set trajectory which is the same for the obstacle but in the opposite direction. Figure 16 shows the trajectory of the vehicle in blue and the one in red is the obstacle: as we can see, the vehicle manages to evade the virtual obstacle and return to the assigned trajectory. The maneuver of obstacle avoidance starts at $t_{\text {start }}$ with the switch to the penalty function of eq. (5) and ends at $t_{O A}$ with the return to the assigned trajectory through eq. (4) till $t_{\text {end }}$.

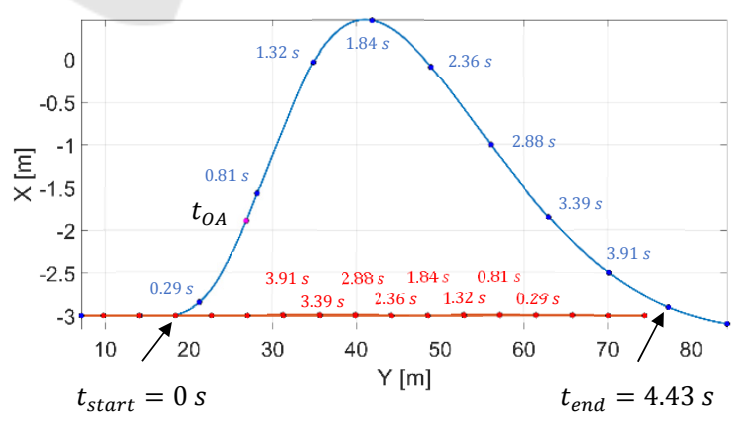

Figure 16: Trajectory of the vehicle in blue and obstacle in red with time.

In the same time period, the evolution of the actuators and longitudinal velocity $u$ are shown. In Figure 17 , the longitudinal velocity decreases a bit while the obstacle avoidance strategy is engaged, then 
the velocity is increasing to the target value which is the speed before the obstacle avoidance intervention.

Alongside this, the throttle shown in Figure 18 is zero when the obstacle is engaged. This happens because the intervention threshold for the obstacle avoidance strategy to intervene is chosen for safety purposes and has a value of 2 s. The control, having enough time, prefers to do a stable maneuver without braking and steering at the same time. Finally, the steering wheel of Figure 19 behaves according to the maneuver depicted in Figure 16.

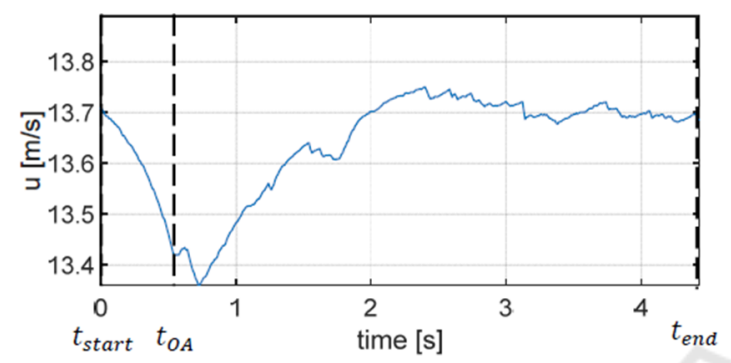

Figure 17: Longitudinal speed between $t_{\text {start }}$ and $t_{\text {end }}$.

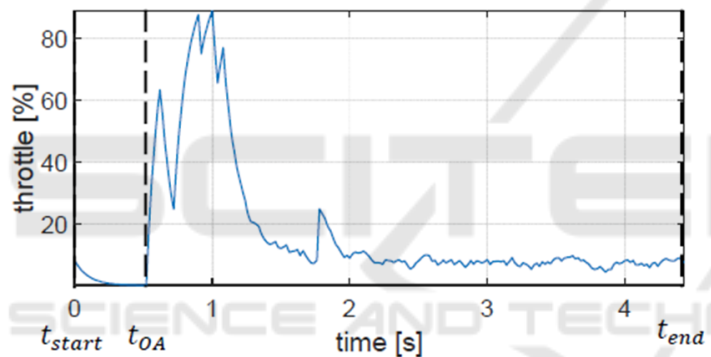

Figure 18: Throttle between $t_{\text {start }}$ and $t_{\text {end }}$.

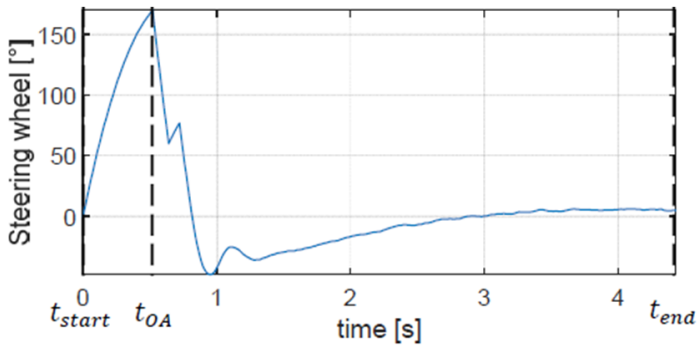

Figure 19: Steering wheel angle between $t_{\text {start }}$ and $t_{\text {end }}$.

\section{CONCLUSIONS}

The Auto Sapiens vehicle, thanks to sophisticated onboard electronics allows the development of custom hardware and software for autonomous vehicles. Currently, the vehicle is being tested with the first ADAS algorithms for obstacle avoidance in case of a frontal crash. The vehicle is able to avoid the obstacle in complete autonomy using Vehicle To Vehicle communication. The entire control system has been developed to begin an experimental campaign aimed at analyzing the performances of the entire system. One of the next steps for future development is related to test the $4 \mathrm{~g}$ technology in preparation for the most promising $5 \mathrm{~g}$ communication network.

\section{REFERENCES}

Antonelli, D., Nesi, L., Pepe, G. and Carcaterra, A. 2018. Mechatronic control of the car response based on VFC. Proceedings of the ISMA2018, Leuven, Belgium, pp. 17-19.

Antonelli, D., Nesi, L., Pepe, G. and Carcaterra, A., 2019a. A novel approach in Optimal trajectory identification for Autonomous driving in racetrack. In 2019 18th European Control Conference (ECC), pp. 3267-3272.

Antonelli, D., Nesi, L., Pepe, G. and Carcaterra, A., 2019b. A novel control strategy for autonomous cars. In 2019 American Control Conference (ACC), pp. 711-716.

Day, C., McEachen, L., Khan, A., Sharma, S. and Masala, G., 2020. Pedestrian recognition and obstacle avoidance for autonomous vehicles using raspberry $\mathrm{Pi}$, Advances in Intelligent Systems and Computing, vol. 1038, pp. 51-69.

Gillmeier, K., Diederichs, F. and Spath, D., 2019, Prediction of ego vehicle trajectories based on driver intention and environmental context. In IEEE Intelligent Vehicles Symposium, Proceedings, vol. 2019-June, pp. 963-968.

Gillmeier, K., Schuettke, T., Diederichs, F., Miteva, G. and Spath, D., 2018. Combined Driver Distraction and Intention Algorithm for Maneuver Prediction and Collision Avoidance. In 2018 IEEE International Conference on Vehicular Electronics and Safety, ICVES 2018.

He, J., He, Z., Fan, B. and Chen, Y., 2020. Optimal location of lane-changing warning point in a two-lane road considering different traffic flows. Physica $A$ : Statistical Mechanics and its Applications, Article vol. 540, Art no. 123000.

Jian, Z., Zhang, S., Chen, S., Lv, X. and Zheng, N., 2019. High-definition map combined local motion planning and obstacle avoidance for autonomous driving. In IEEE Intelligent Vehicles Symposium, Proceedings, vol. 2019-June, pp. 2180-2186.

Laurenza, M., Pepe, G., Antonelli, D. and Carcaterra, A., 2019. Car collision avoidance with velocity obstacle approach: Evaluation of the reliability and performace of the collision avoidance maneuver. In 5th International Forum on Research and Technologies for Society and Industry: Innovation to Shape the Future, RTSI 2019 - Proceedings, pp. 465-470.

Li, M., Li, Z., Xu, C. and Liu, T. 2020. Short-term prediction of safety and operation impacts of lane 
changes in oscillations with empirical vehicle trajectories. Accident Analysis and Prevention, Article vol. 135, 2020, Art no. 105345.

Lynch, K. M. and Frank, C. P. 2017. Modern Robotics: Mechanics, Planning, and Control, 1st ed. MA: Cambridge University Press.

Morales, E. S. et al., 2019. Parallel multi-hypothesis algorithm for criticality estimation in traffic and collision avoidance. In IEEE Intelligent Vehicles Symposium, Proceedings, vol. 2019-June, pp. 21642171.

Pepe, G., Antonelli, D., Nesi, L. and Carcaterra, A., 2018. Flop: feedback local optimality control of the inverse pendulum oscillations. Presented at the ISMA, Leuven.

Pepe, G., Laurenza, M., Antonelli, D. and Carcaterra, A., 2019. A new optimal control of obstacle avoidance for safer autonomous driving. In 2019 AEIT International Conference of Electrical and Electronic Technologies for Automotive (AEIT AUTOMOTIVE), pp. 1-6.

Schraner, M., Bouton, M., Kochenderfer, M. J. and Watzenig, D., 2019. Pedestrian collision avoidance system for scenarios with occlusions. In IEEE Intelligent Vehicles Symposium, Proceedings, vol. 2019-June, pp. 1054-1060.

Song, J., Zhang, W., Wu, X., Cao, H., Gao, Q. and Luo, S. 2019. Laser-based SLAM automatic parallel parking path planning and tracking for passenger vehicle. IET Intelligent Transport Systems, Article vol. 13, no. 10, pp. $1557-1568$,

Taeihagh, A. and Lim, H. S. M., 2019. Governing autonomous vehicles: emerging responses for safety, liability, privacy, cybersecurity, and industry risks. Transport Reviews, vol. 39, no. 1, pp. 103-128.

Ullah, N., Kong, X., Ning, Z., Tolba, A., Alrashoud, M. and Xia, F., 2020. Emergency warning messages dissemination in vehicular social networks: A trust based scheme. Vehicular Communications, Article vol. 22, Art no. 100199.

Xiong, X., Wang, M., Cai, Y., Chen, L., Farah, H. and Hagenzieker, M., 2019. A forward collision avoidance algorithm based on driver braking behavior. Accident Analysis and Prevention, Article vol. 129, pp. 30-43.

www.openstreetmap.org 